EESTI NSV TEADUSTE AKADEEMIA TOIMETISED. XI KÖIDE

FOUSIKALIS-MATEMAATILISTE JA TEHNILISTE TEADUSTE SEERIA. 1962, NR. 4

НЗВЕСТИЯ АКАДЕМИИ НАУК ЭСТОНСКОЙ ССР. ТОМ ХІ

СЕРИЯ ФИЗИКО-МАТЕМАТИЧЕСКИХ И ТЕХНИЧЕСКИХ НАУК. 1962. N. 4

\title{
ТУРБУЛЕНТНАЯ КРУГЛАЯ И ПЛОСКАЯ СТРУИ, РАЗВИВАЮЩИЕСЯ ВО ВСТРЕЧНОМ ПОТОКЕ *
}

\author{
Э. ТИММА
}

До сих пор отсутствуют аналитические работы по турбулентным струям, развивающимся во встречном потоке, которые позволили бы удовлетворительно определить изменение скорости на оси струи.

Экспернментальные исследования Л. А. Вулиса, Т. П. Леонтьевой и В. П. Кашкарова $\left[{ }^{1,2}\right]$, Ю. В. Иванова и Х. Н. Суй $[3,4]$ не охватывают всей длины струи.

Так, в одних работах $[1,2]$ экспериментально. изучено изменение безразмерной дальнобойности $L / d$ круглой струи при значениях параметров $\mu=\frac{u_{\mathrm{H}}}{u_{0 m}}=-0,2 \div-0,48\left(u_{0 m}\right.$ и $u_{\mathrm{r}}-$ соответственно максимальная скорость в начальном сечении струи и скорость потока) и в других $\left[{ }^{3,4}\right]-$ скоростное поле в основном участке круглой и плоской струй в ограниченных пределах изменения определяющего параметра.

Нам неизвестны экспериментальные данные для определения значени'й турбулентных характеристик в начальном участке и в достаточно удаленных сечениях основного участка струи. Совсем не изучены нагретые струи, развивающиеся во встречном потоке.

В настояшей работе сделана попытка экспериментально изучить развитие турбулентных струй, развивающихся во встречном потоке. Эта работа является продолжением исследований, проведенных ранее в Институте энергетики АН ЭССР $\left[{ }^{3,4}\right]$.

Представляется целесообразным вести исследования турбулентной струи, развивающейся во встречном равномерном потоке, по участкам. Можно, например, разделить струю на следующие три характерных участка:

1) начальный участок, на котором имеется потенциальное ядро течения:

2) основной участок, на котором она приобретает такую структуру, что струю можно считать развивающейся из точечного источника или из источника незначительного размера;

3) переходный участок, заключенный между начальным и основным, на котором заканчивается перестройка структуры струи.

Экспериментально исследованы были теперь более подробно начальный и переходный участки и значительно меньше основной участок турбулентной круглой и плоской струй, развивающихся во встречном по-

* Работа выполнена под руководством доктора технических наук Ю. В. Иванова. 
токе. Это обусловлено тем обстоятельством, что основной участок изотермической струи, развивающейся во встречном потоке, более подробно изучен ранее [3,4]. Для проведения опытов была использована экспери ментальная установка, изображенная на фиг. 1, подробное описание которой приведено в предыдущей работе [5].

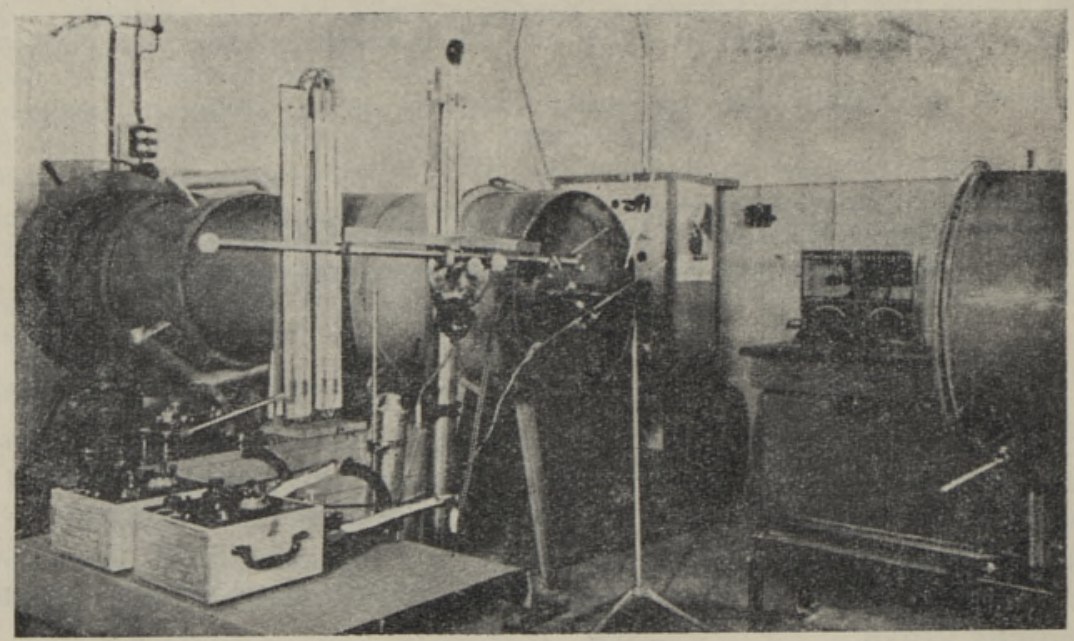

Фиг. 1. Внешний вид установки.

В опытах изменяли скорость струи в пределах от 13 до 20 м/сек, в то время как средняя скорость встречного потока оставалась постоянной и' равной $6,7 \mathrm{~m} /$ сек.

Для образования круглой струи было использовано сопло диаметром 40 мм, а плоской струи - размером $14 \times 200$ мм (с ориентацией перпендикулярно большей стороне). Максимальная разность температур между ненагретой струей и потоком не превышала 8 градусов, и поэтому можно считать, что отношение максимальной абсолютной температуры струи $\left(T_{0 m}\right)$ к абсолютной температуре потока $\left(T_{\text {н }}\right) \Theta=T_{0 m} / T_{\text {н }}$ примерно равняется единице. Температура потока на протяжении опытов равнялась комнатной.

В сечениях струи измерены скорость в направлении оси струи и температура $T$ при следующих значениях параметров:

1. Для плоской струи

а) в изотермической струе при $\Theta \approx 1$ и отношениях скоростей потока и струи $\mu=\frac{u_{\mathrm{H}}}{u_{0 m}}=-0,33 ;-0,50$.

б) в неизотермической струе при $\Theta=1,48$ и $\mu=-0,3$.

2. Для круглой струи

а) в изотермической струе $(\Theta=1)$ при $\mu=-0,33 ;-0,50$.

б) в неизотермической струе при $\Theta=2$ и $\mu=-0,33$. Так как отнсшение диаметров потока и сопла у нас сравнительно большое, то согласно формуле, приведенной ранее [4], можно установить, что при значении параметра $\mu=-1 \div-0,3$ струя развивается во встречном потоке, но при $\mu>-0,3$ происходит взаимодействие струи не с потоком, а со встречной струей. 
Температурные и скоростные поля в сечени'я струи оказались симметричными, поэтому можно было уменьшить число измерений. Для определения скоростного или температурного полей в сечени'ях круглой струи было достаточно провести измерения по горизонтальной линии; проходящей через центр. На фиг. $2 a$ и б показаны скоростные профили в разных сечениях круглой струи при $\Theta=1$ и $\lambda=\frac{1}{\mu}=-3,-2$ и плоской струи при $\Theta=1$ и $\lambda=-2$. Из этих фигур видно, что при указанных значениях параметров в начальном сечении струи имеется заметная деформация скоростного поля потока.

Относительная ошибка при' измерении скоростей в начальном и переуодном участках струй не превышала $3 \%$. Причем относительная точность была больше у оси струи и уменьшалась в направлении внешних границ струи.

Вблизи линии нулевой скорости относительная ошибка больше и увеличиваегся с удалением от сопла. Вблизи точки на оси, где скорость $u=0$, отмечались пульсании скорости, которые заметно затрудняли измерения. Можно сказать, что пульсации скорости' увеличиваются в конне основного участка струи. При измерениях температур относительная ошибка не превышала $4 \%$.

Известно, что одним важнейшим свойством турбулентной струи, развивающейся в спутном потоке или неподвижной среде, является подобие профилей скоростей в начальном, переходном и основном участках. Под подобием профилей подразумевается возможность представить скоростные профили в разных сечениях струй при помощи.одной и той же функщии

$$
\frac{u-u_{\mathrm{H}}}{u_{\mathrm{s}}^{\prime}-u_{\mathrm{H}}}=f_{k}(\eta)
$$

где $u, u_{\mathrm{H}}, u_{m}$ - скорости соответственно в произвольной точке струи, в потоке и на оси струи;

$f_{k}(\eta)$ - универсальная функция в сечениях струи, могущая иметь разный вид в начальном, переходном и основном участках;

$\eta$ - безразмерная ордината точки, определяющаяся в начальном участке струи формулой

$$
\eta=\frac{y-y_{1}}{v_{2}-y_{1}}
$$

а в переходном и основном участках струи -

$$
\eta=\frac{y}{\delta}
$$

причем $y$ - расстояние точки от оси струи;

$y_{1}$ и $y_{2}$ - ординаты точки, соответственно внутренней и внешней границы зоны смешения в начальном участке струи;

$\delta$ - ордината границы струи в переходном и основном участках. Для того, чтобы проверить упомянутое выше подобие профилей турбулентной струи, развивающейся во встречном потоке, надо рассчитать, что в формуле (1) скорость встречного потока $u_{\text {н }}$ является отрицательной. Так как экспериментальные определения граничной точки, где 

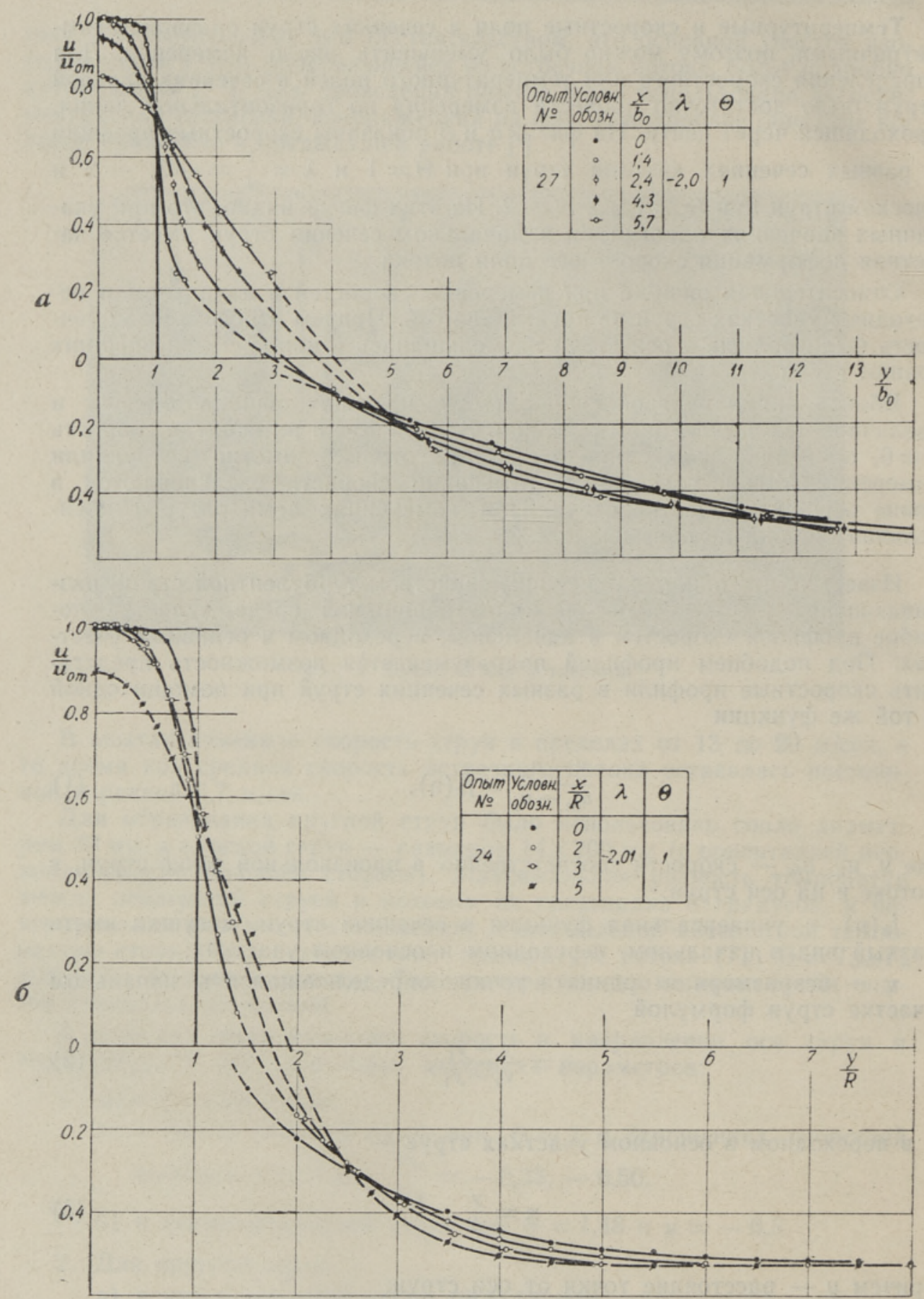

Фиг. 2. Профили скорости в различных сечениях турбулентной струи, развивающейся во встречном потоке: $a$ - плоская струя; $\sigma-$ круглая струя.

безразмерная скорость $u-u_{\text {н }}$ равна нулю, возможны только с большой ошибкой, экспериментальные данные представлены на фиг. 3 в следующих координатах: 


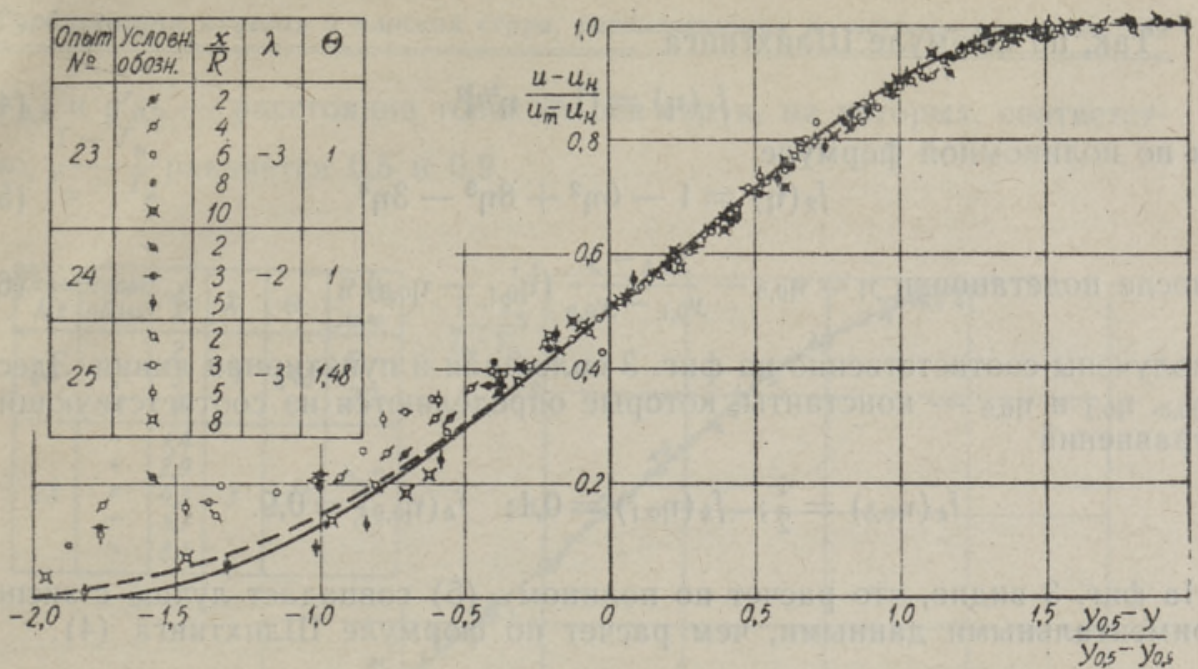

\begin{tabular}{|c|c|c|c|c|c|c|c|c|}
\hline \multirow{2}{*}{$\begin{array}{c}\text { Onblm } \\
N 2\end{array}$} & \multirow{2}{*}{$\lambda$} & \multirow{2}{*}{$\theta$} & \multicolumn{6}{|c|}{ Paccmoянuе no ocu $x(\mu, \mu)$} \\
\hline & & & 10 & 20 & 30 & 40 & 60 & 100 \\
\hline 26 & -3 & 1 & $\cdot$ & $\Delta$ & a & $\times$ & . & $\bullet$ \\
\hline 27 & -2 & 1 & 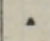 & ? & 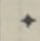 & म4 & x & - \\
\hline 28. & -3 & 1,48 & - & ? & $\rightarrow$ & क & 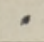 & \& \\
\hline
\end{tabular}
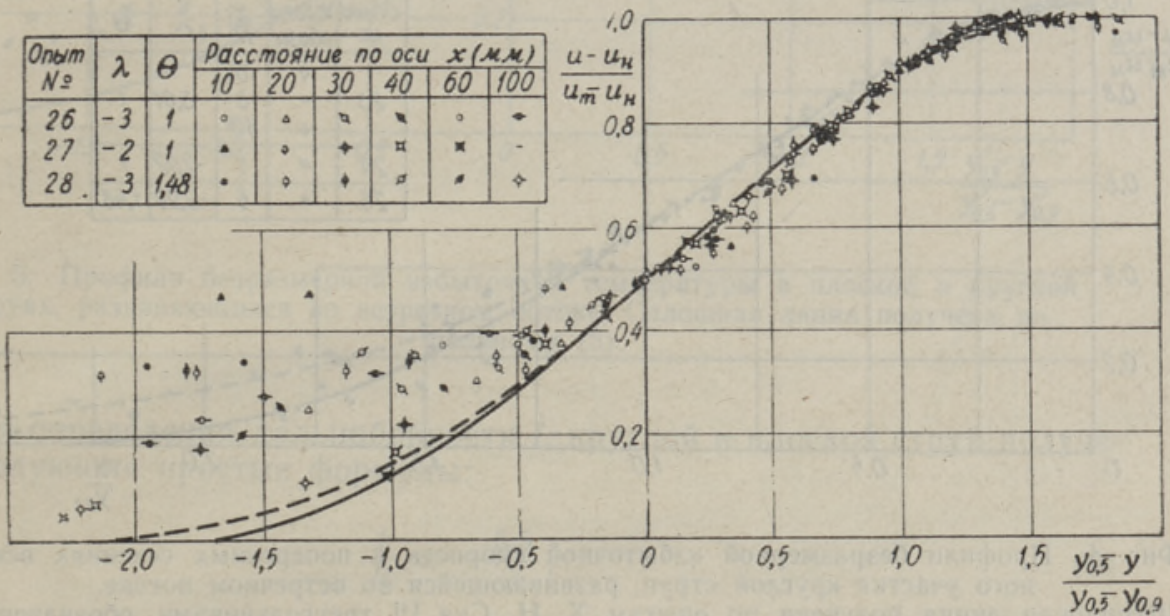

Фиг. 3. Профили безразмерной избыточной скорости в поперечных сечениях турбулентной струн, развивающейся во встречном потоке: $a$ - круглая струя; б - плоская струя.

$$
\frac{u-u_{\mathrm{H}}}{u_{m}-u_{\mathrm{H}}} \quad \text { и } \quad \frac{y_{0,5}-y}{y_{0,5}-y_{0,9}},
$$

где $y_{0,5}, y_{0,9}$ - ординаты точки и соответственно безразмерная избыточная скорость $\frac{u-u_{\text {н }}}{u_{m}-u_{\text {н }}}$ равняется 0,5 и 0,9. Простое видоизменение показывает, что если экспериментальные данные ложатся на одну кривую, то выполняется условие подобия (1).

Из изучения подобия профилей скорости $\frac{u-u_{\text {н }}}{u_{m}-u_{\mathrm{H}}}$ в различных сечениях струй следует, как видно на фиг. 3, что подобие в начальном и переходном участках круглых и плоских струй наблюдается только в пределах значения безразмерной скорости от 0,3 до 1. Для определения профилей можно использовать формулы, приведенные в предыдущей работе [5]. 
Так, по формуле Шлихтинга

$$
f_{1}(\eta)=\left[1-\eta^{3 / 2}\right]^{2}
$$

и по полиномной формуле

$$
f_{2}(\eta)=1-6 \eta^{2}+8 \eta^{3}-3 \eta^{4}
$$

после подстановки $\eta=\eta_{0,5}-\frac{y_{0,5}-y}{y_{0,1}-y_{0,9}}\left(\eta_{0,1}-\eta_{0,9}\right)$

получены соответственно на фиг. 3 сплошная и пунктирная линии. Здесь $\eta_{0,5}, \eta_{0,1}$ и $\eta_{0,9}-$ константы, которые определяются из соответствующих уравнений

$$
f_{k}\left(\eta_{0,5}\right)=\frac{1}{2} ; \quad f_{k}\left(\eta_{0,1}\right)=0,1 ; \quad f_{k}\left(\eta_{0,9}\right)=0,9
$$

На фиг. 3 видно, что расчет по полиному (5) совпадает лучше с экспериментальными данными, чем расчет по формуле Шлихтинга (4).

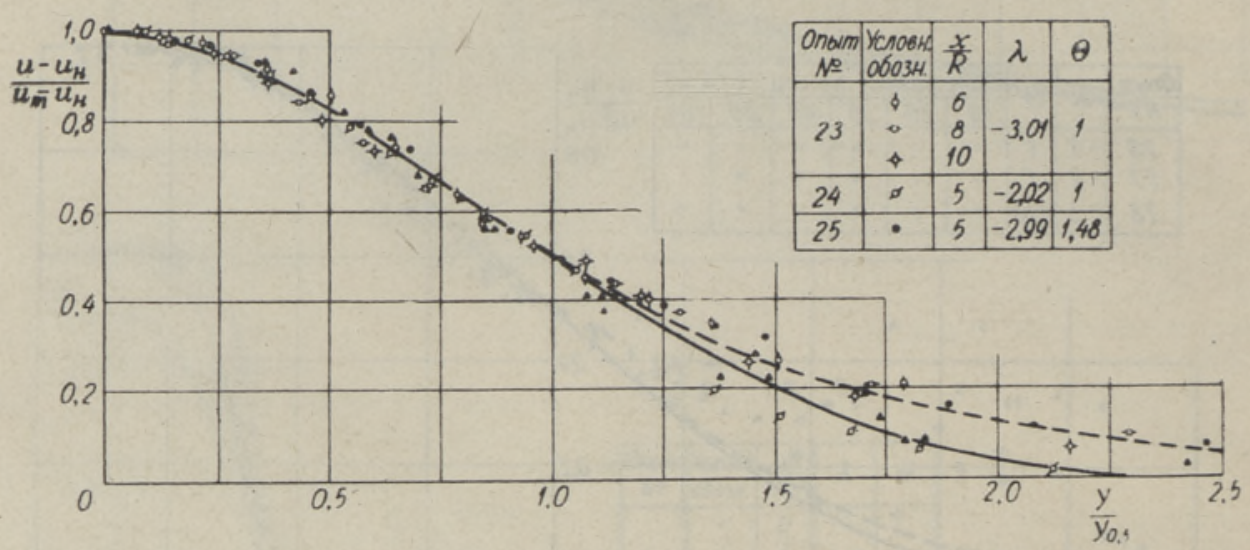

Фиг. 4. Профили безразмерной избыточной скорости в поперечных сечениях основного участка круглой струи, развивающейся во встречном потоке.

Штирховая линия получена по опытам Х. Н. Суя [4], треугольниками обозначенные профили получены по опытам Л. А. Вулиса [1] и сплошная линия получена по полиному (5).

На фиг. 4 приведено сравнение профилей безразмерной избыточной скорости в разных сечениях круглой струи по опытам Х. Н. Суя $\left.{ }^{4}\right]$, Л. А. Вулиса ['] и автора. Видно, что в основном участке турбулентной круглой струи, развивающейся во встречном потоке, профили скорости подобны и их можно удовлетворительно определить при помощи полинома (5) подстановкой

$$
\eta=\eta_{0,5} \frac{y}{y_{0,5}} .
$$

На фиг. 5 приведен температурный профиль струи в безразмерных координатах. Там видно, что профили безразмерной избыточной температуры $\frac{T-T_{\text {н }}}{T_{m}-T_{\text {н }}}$ в разных сечениях круглой и плоской струй подобны и могут быть расчитаны по формуле (5).

$T, T_{H}, T_{m}$ - температура, соответственно в произвольной точке струи, в потоке и на оси струи. 
$y_{0,5}^{\prime}$ и $y_{0.9}^{\prime}$ - расстояния точек от оси струи, на которых, соответственно, $\frac{T-T_{\text {н }}}{T_{m}-T_{\text {н }}}$ равняется 0,5 и 0,9 .

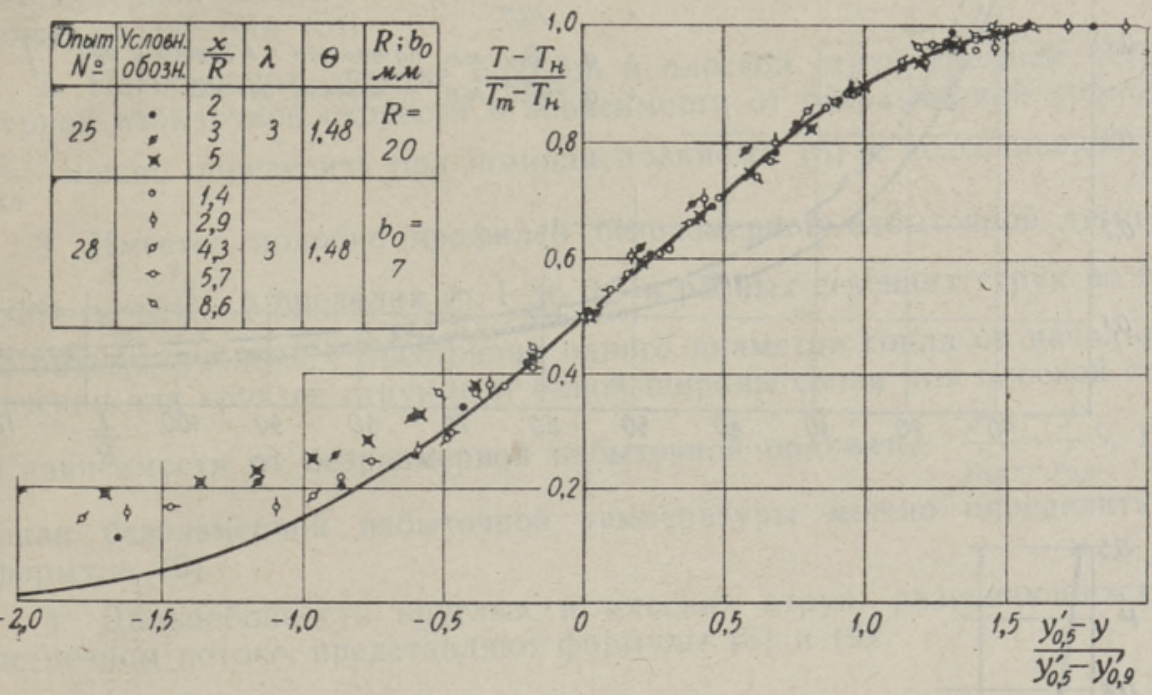

Фиг. 5. Профили безразмерной избыточной температуры в плоской и круглой струях, развивающихся во встречном потоке. Сплошная линия получена по полиному (5).

Для определения дальнобойности $L$ круглой и плоской струй получены следующие простые формулы:

$$
\begin{gathered}
\frac{L}{R_{\mathrm{t}}}=-\frac{5,5}{\mu}, \\
\frac{L}{v_{0}}=\frac{1-28 \mu}{\mu^{2}}-47,
\end{gathered}
$$

где $R$ - радиус круглого сопла;

$b_{0}$ - полуширина плоского сопла.

На фиг. $6 a$ приведено сравнение опытных данных с расчетом по формуле Х. Н. Суя [4], Л. А. Вулиса $\left[{ }^{1,2}\right]$ и формулой (8). В формуле Л. А. Вулиса

$$
\frac{L}{R}=\frac{2 k}{|\mu|}
$$

где коэффициент $k=3 \div 3,5$.

При сравнении расчетов на фиг. $6 a$ принято осредненно

$$
k=3,25 \text {. }
$$

На фиг. $6 a$ пунктирная линия проведена по формуле (8), штриховая линия по формуле (8a) и сплошная линия по формуле Суя [4]. Как видно из фиг. $6 a$, лучшее совпадение дает формула (8). 

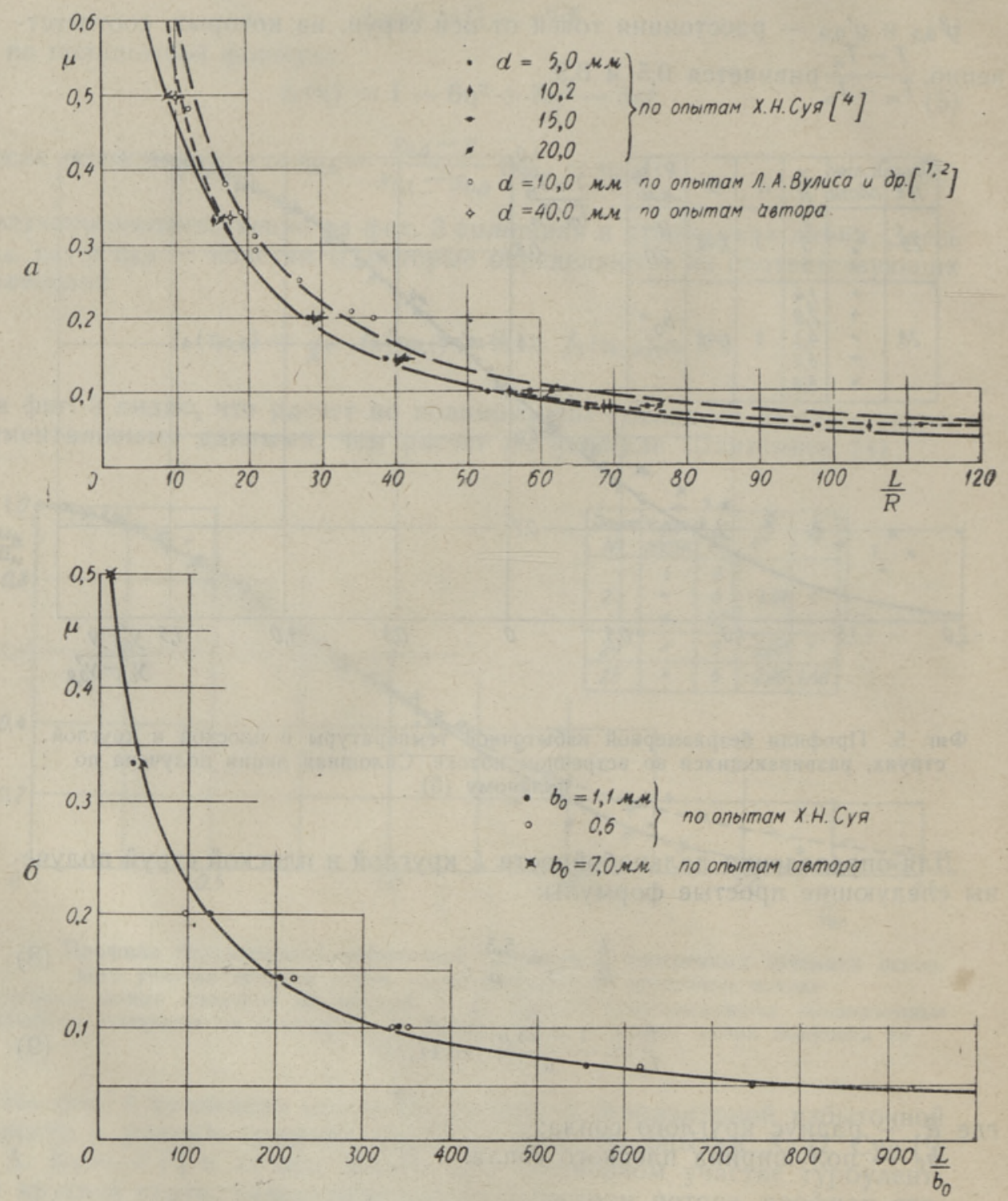

Фиг. 6. Сравнение опытных данных по дальнобойности струй с расчетом по формулам различных авторов:

$a$ - круглая струя; пунктирная линия получена по формуле (8), сплошная линия - по формуле X. Н. Суя [4] и штриховая линия - по формуле Л. А. Вулиса (8a).

6 - плоская струя; сплошная линия получена по формуле (9).

На фиг. 66 видно, что сплошная линия, проведенная по формуле (9), хорошо описывает опытные данные в пределах изменения параметра $\mu$ от 0 до $-0,5$.

\section{Выводы}

1. Имеется подобие профилей безразмерной избыточной скорости $\frac{u-u_{\text {н }}}{u_{m}-u_{\text {н }}} \quad$ в пределах от 1 до 0,3 в разных сечениях струи во всем ее 
объеме, начиная с расстояния одного диаметра сопла от начального сечения для круглой струи и от одной ширины сопла для плоской струи в зависимости от безразмерной избыточной ординаты $\frac{y_{0,5}-y}{y_{0,5}-y_{0,9}}$. Профили безразмерной избыточной скорости можно определить при помощи формулы (4) или (5).

2. На основном участке круглой и плоской струй профили безразмерной и'збыточной скорости в зависимости от безразмерной ординаты $\frac{y}{y_{0,5}}$ можно определить при помощи полинома (5) с подстановкой (7).

3. Имеется подобие профилей безразмерной избыточной температуры $\frac{T-T_{\text {н }}}{T_{m}-T_{\text {н }}}$ в пределах от 1 до 0,3 в разных сечениях струи во всем ее объеме, начиная с расстояния одного диаметра сопла от начального сечения для круглой струи и от одной ширины сопла для плоской струи в зависимости от безразмерной избыточной ординаты $\frac{y_{0,5}^{\prime}-y}{y_{0,5}^{\prime}-y_{0,9}^{\prime}}$. Профн'ли безразмерной избыточной температуры можно определить по формуліе (5).

4. Дальнобойность круглых и плоских струй, развивающихся во встречном потоке, представляют формулы (8) и (9).

\section{ЛИТЕРАТУ РА}

1. Л. А. Вулис и Т. П. Л еон ть в в а, О спутных и встречных турбулентных струях, Изв. АН Қаз. ССР, сер. энергетика, вып. 9, 1955.

2. Л. А. Вулис, В. П. Кашкаров и Т. П. Л еонтьев а, Исследование сложных турбулентных струйных течений. Исследование физических основ рабочего процесса топок и печей, под ред. Л. А. Вулиса, Изд. АН Каз. ССР, 1957.

3. Х. Н. С уй, Ю. В. Ив анов, Исследование развития круглой струи в начальном участке встречной струи большего размера, Изв. АН ЭССР, сер. техн. и физ.-мат. наук, т. VIII, № 2, 1959.

4. Х. Н. С у й, Исследование развития круглой и плоской струй во встречном и спутном потоке, Изв. АН ЭССР, сер. физ.-мат. и техн. наук, т. Х, № 3, 1961.

5. Э. П. Тимма, Ю. В. Ив анов, Круглая и плоская турбулентные струи, развивающиеся в спутном потоке, Изв. АН ЭССР, сер. физ.-мат. и техн. наук, т. XI, № 3, 1962 .

Институт энергетики

Академии наук Эстонской ССР

Поступила в редакцию

5. III 1962

\section{OMARA JA LAMEDA JOA LEVIMINE VASTUVOOLUSES}

\section{E. TIMMA}

\section{Resümee}

Käesolevas töös on eksperimentaalselt uuritud turbulentset vastuvooluses levivat lamedat ja ümarat juga. Katseandmete põhjal selgitatakse suhtelise kiiruse $\frac{u-u_{\mathrm{H}}}{u_{m}-u_{\mathrm{H}}}$ ja suhtelise temperatuuri $\frac{T-T_{\mathrm{B}}}{T_{m}-T_{\mathrm{H}}}$, profiilide sarnasuse küsimust joa erinevates ristlöigetès 
ning esitatakse valemid nende profiilide määramiseks. Vastuvooluses areneva turbulentse ümara ja lameda joa ulatuvuse määramiseks on leitud valemid, mida on vōrreldud mitmete autorite eksperimentaalandmetega.

\section{Eesti NSV Teaduste Akadeemia Energeetika Instituut}

Saabus toimetusse

5. III 1962

\section{DEVELOPMENT OF A ROUND AND A FLAT JET IN A TRANSVERSE STREAM}

\section{E. Timma}

\section{Summary}

An experimental study has been made of turbulent flat and round jets developing in a transverse stream. On the basis of experimental data the problem of the similarity of the profiles of relative velocity. $\frac{u-u_{\mathrm{H}}}{u_{m}-u_{\mathrm{H}}}$ and temperature $\frac{T-T_{\mathrm{H}}}{T_{m}-T_{\mathrm{H}}}$ in different crosssections of the jet has been elucidated, and formulas have been presented for computing these profiles. Corresponding formulas have been found for determining the extension of turbulent round and flat jets in a transverse stream, and have been compared with the experimental data of various authors.

Academy of Sciences of the Estonian S.S.R. Institute of Energetics

Received March 5th, 1962 